\title{
$\bullet$ \\ Haematological Findings and Pattern Analysis in Patients with COVID-19 Infection
}

\section{IJCRR}

Section: Healthcare ISI Impact Factor (2019-20): 1.628

IC Value (2019): 90.81 $\operatorname{SJIF}(2020)=7.893$

(c) (i) (3)

Copyright@IJCRR

\section{Piyush Sahu' ${ }^{1}$ Anshika Rai', Shilpi Sahu ${ }^{3}$, Kishor Raut ${ }^{4}$}

\begin{abstract}
'Primary Investigator, Assistant Professor, Department of Pathology, MGM Medical College and Hospital, Sector-1, Kamothe, Navi Mumbai410209, Maharashtra, India; ${ }^{2}$ Co-lnvestigator, 3 rd-year Resident, Department of Pathology, MGM Medical College and Hospital, Sector-1, Kamothe, Navi Mumbai- 410209, Maharashtra, India; ${ }^{3}$ Co-lnvestigator, Professor and Head, Department of Pathology, MGM Medical College and Hospital, Sector-1, Kamothe, Navi Mumbai- 410209, Maharashtra, India; ${ }^{4}$ Co-lnvestigator, Statistician, Department of Community Medicine, MGM Medical College and Hospital, Sector-1, Kamothe, Navi Mumbai- 410209, Maharashtra, India.
\end{abstract}

\section{ABSTRACT}

Introduction: The coronavirus disease 2019 (COVID-19) is caused by the virus SARS-CoV-2 and is declared as a global pandemic by the World Health Organization (WHO). Recently, many western and Chinese studies have done, but there is a need for research to evaluate the pattern of the haematological parameters of COVID-19 patients in the Indian population.

Objective: The objective is to study the haematological parameters which include haemoglobin, Platelet count, Total leucocyte count, differential count, Neutrophil: Lymphocyte ratio (NLR), Platelet: Lymphocyte ratio (PLR) and Peripheral smear findings and to observe the correlation between various parameters and establish a pattern in Intensive care unit (ICU) and Non-ICU set-up in the Indian scenario.

Methods: A retrospective study carried out on 150 COVID-19 patients admitted in June and July 2020 in a tertiary care hospital, Navi Mumbai, India. The haematological parameters of both Non-ICU and ICU patients were studied and compared for statistical analysis.

Results: We observed lymphopenia, eosinopenia, neutrophilia, leucocytosis and decline of haemoglobin in COVID-19 confirmed cases. These parameters showed significant aggravation in critically-ill ICU patients as compared to the non-ICU group. Increased NLR identified as an important risk factor of severe illness. Although PLR did not show any significant difference. Morphology of leucocytes showed reactive changes.

Conclusion: Real-time Polymerase chain reaction (RT-PCR), the confirmatory diagnostic test of COVID-19 is time-consuming and less accessible. Therefore, for initial diagnosis, severity assessment and ICU requirement, these haematological parameters are valuable.

Key Words: Coronavirus, COVID-19, Neutrophil: Lymphocyte ratio, Platelet: Lymphocyte ratio, Complete blood cell count

\section{INTRODUCTION}

Today the world is facing a pandemic, caused by a novel virus, Severe acute respiratory syndrome (SARS) coronavirus (SARS-CoV). ${ }^{1}$ The Clinical characterization of COVID-19 has been broadly defined by $\mathrm{WHO}^{2}$ with most of the confirmed COVID-19 cases have mild to the moderate clinical presentation which can rapidly deteriorate threatening life. The diagnosis of COVID-19 is confirmed by RT-PCR, which has limited availability, variable turnaround time, and low sensitivity. Basic haematological indicators can be readily obtained from a routine complete blood cell count (CBC), is inexpensive and may provide prognostic information thereby lowering the mortality rate..$^{1-3}$
The National Health Commission of China, ${ }^{3}$ WHO interim Guidance $^{2}$ as well as many Chinese and western studies currently recommend many haematological parameters including Neutrophil/lymphocyte ratio (NLR) and platelet/lymphocyte ratio may also have prognostic value in determining severity. However, there is a need for research to evaluate the pattern of the haematological parameters of COVID-19 patients in the Indian population., ${ }^{4}$ In the present study we aimed to study the haematological parameters in COVID-19 patients, which includes haemoglobin, RBC indices, Platelet count, Total leucocyte count, differential count, Neutrophil: Lymphocyte ratio (NLR), Platelet: Lymphocyte ratio (PLR) and Peripheral smear findings. In addition, we will establish the correlation between various haematological parameters.

\section{Corresponding Author:}

Dr. Piyush Sahu, Assistant Professor Department of Pathology, MGM Medical College and Hospital, Sector-1, Kamothe, Navi Mumbai- 410209, Maharashtra, India; Mobile: +91 7977556844

ISSN: 2231-2196 (Print)

Received: 10.09 .2020
ISSN: 0975-5241 (Online)

\author{
Revised: 13.11 .2020
}

Accepted: 18.12 .2020

Published: 20.03 .2021 


\section{MATERIAL AND METHODS}

A retrospective study carried out on 150 COVID-19 positive patients admitted in June and July 2020 in a tertiary care hospital, Navi Mumbai, India. Institutional Ethics committee approval was obtained. The positive cases of COVID-19 by RT-PCR were included in the study. Patients with chronic lung diseases, haematological disorders and malignancy on treatment were excluded from the study. In this study, for both Non-ICU and ICU patients, the first blood sample collected for obtaining the haematological parameters was considered and compared for statistical analysis. ${ }^{6-8}$

$\mathrm{CBC}$ was done on 7-part haematological analyzer (Sysmex $\mathrm{XN}-1000$ ). It includes following parameters with their respective cut-off values: Hemoglobin: $13.5-17.5 \mathrm{~g} / \mathrm{dl}$ (male) and $12.0-15.6 \mathrm{~g} / \mathrm{dl}$ (female); Platelet count: $1.5-4.5 \mathrm{lakh} /$ cumm; Total WBC count: 4,000 - 11,000/cumm, Absolute neutrophil count (ANC):1,500- 8,000/cumm; Absolute lymphocyte count (ALC):1,000 - 4,800/cumm; Neutrophil count: 55-70\%; Lymphocyte count: 20-40\%; Eosinophil count: 0-6\%; Monocyte count: $0-7 \%$.

Neutrophil lymphocyte ratio (NLR) was calculated by taking the ratio of absolute neutrophil count to absolute lymphocyte count with cut-off $<3.13$. Platelet Lymphocyte ratio (PLR) was calculated by taking the ratio of platelet count to absolute lymphocyte count with cut-off $<180$. Peripheral blood smears were stained by Romanowsky stains to study the Red Blood Cell, White Blood Cell and Platelets morphology. These parameters were assessed and compared in both the groups i.e. Non-ICU and ICU groups and a pattern was established to predict the prognosis.

The Statistical analysis was done using SPSS 21 software. The test of significance was established at the level of significance of 0.01 or 0.05 . For comparing the mean and standard deviation (SD), an independent t-test was used.

\section{RESULTS}

\section{Demographic characterization}

Out of the 150 COVID-19 patients, 109 cases (72.6\%) were admitted in the wards (Non-ICU) and 41 cases $(27.3 \%)$ in ICU. The common clinical presentation in the Non-ICU group was fever, cough, sore throat and myalgia. While, those from the ICU group were having dyspnea, respiratory distress and shock. There was a significant difference in the median age between the two groups $(\mathrm{P}<0.01)$. Of the total 150 patients, 105 were male $(70 \%)$ and 45 were female $(30 \%)$. There was no significant difference in the gender composition between the two groups of patients $(\mathrm{P}>0.01)$ shown in Table 1.

Table 1: Haematological and other blood cell counting findings

\begin{tabular}{|c|c|c|c|c|c|c|}
\hline \multirow[t]{2}{*}{ Parameters } & \multicolumn{2}{|c|}{ NON- ICU } & \multicolumn{2}{|l|}{ ICU } & \multirow[t]{2}{*}{ P-Value } & \multirow[t]{2}{*}{ Test significance } \\
\hline & Mean & SD & Mean & SD & & \\
\hline Age & 38.8 & 13.8 & 52.4 & 16.3 & $<0.01$ & significant \\
\hline Hemoglobin & 12.7 & 2.3 & 11.5 & 2.1 & $<0.01$ & significant \\
\hline RBC count & 4.6 & 0.8 & $4 \cdot 3$ & 0.8 & $>0.01$ & Non-significant \\
\hline PCV (Hematocrit) & 38.7 & 6.3 & $35 \cdot 9$ & 6.2 & $>0.01$ & Non-significant \\
\hline MCV( mean carcuscular volume) & $84 \cdot 9$ & 10.2 & $84 \cdot 4$ & 8.1 & $>0.01$ & Non-significant \\
\hline MCH(mean carcuscular haemoglobin) & $27 \cdot 7$ & 4.2 & 27.1 & 2.7 & $>0.01$ & Non-significant \\
\hline $\begin{array}{l}\text { MCHC(mean carcuscular haemoglobin } \\
\text { concentration) }\end{array}$ & 32.5 & 1.6 & 32.2 & 1.6 & $>0.01$ & Non-significant \\
\hline RDW ( red cell distribution width) & 14.6 & 2.2 & $15 \cdot 4$ & 2.8 & $>0.01$ & Non-significant \\
\hline Total WBC count & 6421.6 & $2805 \cdot 5$ & 11170.7 & 4443.8 & $<0.01$ & significant \\
\hline Neutrophils \% & 60.6 & 11.7 & 82.0 & 8.7 & $<0.01$ & significant \\
\hline Absolute neutrophil count & $4023 \cdot 5$ & $2375 \cdot 3$ & 9321.5 & 4190.3 & $<0.01$ & significant \\
\hline Lymphocytes $\%$ & 34.0 & 11.1 & 13.2 & 8.0 & $<0.01$ & significant \\
\hline Absolute lymphocyte count & 2068.0 & 894.8 & 1305.8 & 701.4 & $<0.01$ & significant \\
\hline Monocytes count & $4 \cdot 3$ & 1.6 & 3.9 & 1.9 & $>0.01$ & Non-significant \\
\hline Eosinophil count & 1.3 & 1.7 & 0.6 & 1.0 & $<0.01$ & significant \\
\hline Platelet count & 200100.9 & 78277.8 & 186414.6 & $75327 \cdot 9$ & $>0.01$ & Non-significant \\
\hline Neutrophil: Lymphocyte Ratio & 2.1 & 1.2 & 10.4 & 10.3 & $<0.01$ & significant \\
\hline Platelet: Lymphocyte Ratio & 107.9 & 50.3 & $199 \cdot 3$ & 222.4 & $>0.01$ & Non-significant \\
\hline
\end{tabular}


In our study, as shown in Table 1, the following significant findings were observed.

Mild anaemia was noted in both groups, also there was a significant difference in the mean haemoglobin levels. There were no significant difference found in RBC count, RBC indices (MCV, $\mathrm{MCH}$ and $\mathrm{MCHC}$ ) and Red cell distribution width. Our study showed significantly raised total leukocyte count in the ICU group as compared to the non-ICU group. In Differential WBC count, Neutrophilia was significantly observed in the ICU group, while it was in the normal range in the Non-ICU group. Both Neutrophil count as well as absolute neutrophil count seem to increase according to the severity of the COVID-19. One of the important findings in this study was, decreased lymphocyte count (lymphopenia) which was observed in the ICU group more as compared to the Non-ICU group, suggesting that it serves as an important prognostic indicator. Another important finding was a significantly low Eosinophil count in the ICU group. In many critically ill cases, eosinophil count was found to be 0 . There were no significant differences in Monocyte count in both groups. And since the Basophil count was 0 in all the patients, no comparison could be made. Neutrophil: Lymphocyte ratio was found to be significantly raised in ICU patients as compared to Non-ICU patients. ${ }^{10-12}$

There was no significant difference observed in platelet count and Platelet: lymphocyte ratio. Significant Peripheral smear findings were seen in a few ICU cases (Figure 1). In 2 cases, Neutrophils showed C-shaped fetus-like nuclei, 4 ICU cases showed toxic granules and toxic vacuolization. In 5 cases, lymphocytes showed reactive changes in the form of scalloping edges and cytoplasmic vacuoles. Monocytes also showed reactive changes in 2 cases. Two ICU cases showed leucoerythroblastic reaction in the form of raised nucleated RBCs and occasional myeloid series precursor. RBC morphology in both ICU and non-ICU patients were mostly normocytic normochromic followed by microcytic hypochromic. Platelets were normal in size and morphology.

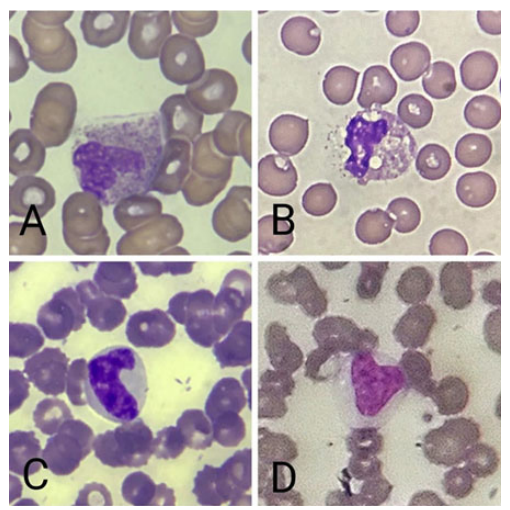

Figure 1: Morphology of leucocytes: A- Toxic granulation in neutrophil, B- Toxic vacuolization in neutrophil, C- Fetus shaped nuclei in a myeloid precursor cell, D- Reactive lymphocyte.

\section{DISCUSSION}

\section{Haemoglobin and RBC indices:}

In SARS-CoV-2 infection, Anemia was not a common laboratory finding of patients ${ }^{4}$. However, a meta-analysis by Lippi and Mattiuzzi ${ }^{5}$ showed that patients with severe symptoms showed lower haemoglobin levels compared to those with mild symptoms. The first COVID-19 case in the USA showed a slight decrease in haemoglobin on day 6 and then as the patient recovered, Hemoglobin levels also improved. ${ }^{6}$ In a study reported by Jin Yin-tan Hospital, Hemoglobin was below the normal range in patients with SARS-CoV-2 infection. ${ }^{7}$ In another study of 1099 patients with COVID-19, the haemoglobin in the severe group showed a lower value compared to that in the non-severe one ${ }^{8}$. In the report of Zhou et al., the haemoglobin of the patients with severe cases decreased more significantly. ${ }^{9}$

A decrease in haemoglobin is seen in patients of SARSCoV-2 infection because inflammatory changes interfere with erythropoiesis. The main cytokines which are increased are IL-1, IL-6, INF- $\gamma$ and TNF- $\alpha^{10}$. IL-1 reduces RBC count by neocytolysis, ${ }^{11}$ TNF- $\alpha$ and IL-1 inhibit erythropoietin (EPO) production by forming reactive oxygen species, ${ }^{12}$ INF- $\gamma$ down-regulates EPO receptor expression and causes apoptosis of erythrocyte progenitor cells ${ }^{13}$ and IL-6 impairs haemoglobin production and erythroid cell maturation. ${ }^{14}$ Thus, reduced haemoglobin levels might be an indicator of disease progression in COVID-19 patients.

\section{Total and Differential WBC count}

Coronavirus infection is associated with alterations in the white blood cell count. Our study showed similar results as in previous study. ${ }^{15}$ Other recent studies exhibited that COVID-19 patients were more likely to have normal TLC., ${ }^{4,16-18}$ But few studies also suggested Leucopenia as predominant findings in critically-ill ICU patients. ${ }^{8}{ }^{819}$ A study from Wuhan reported no marked change in WBC counts, lower severity of symptoms and a full recovery, particularly in adults with no co-morbidities. ${ }^{20}$ So basically, TLC has very diverse findings in literature when compared severe and non-severe patients. The mechanism of this phenomenon is not fully understood, although some authors hypothesize that it is associated with the intensification of the cytokine storm syndrome ${ }^{20}$ which is a clinical state of hyper inflammation due to the release of proinflammatory cytokines and chemokines. Cytokine storm syndrome may result in multiorgan failure, which can be fatal. ${ }^{21,22}$

In Differential WBC count, Neutrophilia was significantly observed in the ICU group and it showed an increase according to the severity of the COVID-19. It was observed in both ANC and neutrophil percentage. A significant difference in median ANC of ICU and non-ICU patients were 
found in similar studies done at Wuhan Zhongnan Hospital, ${ }^{23}$ Beijing Ditan Hospital ${ }^{24}$ as well as in a Tertiary care hospital in Singapore. ${ }^{25}$ According to another retrospective study from Wuhan, China, patients with ARDS developed higher neutrophils than those without ARDS. ${ }^{26}$ The neutrophilia in ICU patients could be due to cytokine storm or attributed to excessive inflammation, immunosuppression and sepsis.

Secondly, Lymphopenia was characteristically demonstrated in critically-ill ICU patients compared to Non-ICU patients in our study as well as many other studies. ${ }^{23,26-30}$ The mechanism of this phenomenon is not fully understood, although some authors hypothesize that it is associated with the intensification of the inflammatory process and/or direct infection of lymphocytes and destruction of lymphoid organs. ${ }^{21,31}$ Molecular studies also suggest that the virus can infect $\mathrm{T}$ cells through receptor-dependent, $\mathrm{S}$ protein-mediated membrane fusion $^{32}$, which results in the reduction of the cytotoxic capacity of lymphocytes..$^{33}$ Additionally, patients treated with glucocorticoids were also known to cause lymphopenia. ${ }^{34,35}$

Another important finding was significantly low or completely absent Eosinophil count in the ICU group. A study was done by Fahmina Tanni, Eleonora Akker, et al. ${ }^{36} \mathrm{com}-$ pared COVID-19 patients and the influenza group. It showed significant numbers of patients in the COVID-19 group had an eosinophil count of 0 . Their data showed that improvement in and recovery of eosinophil counts corresponds to a better prognosis. ${ }^{36}$ Our findings are also consistent with a recent report from China. ${ }^{37}$ The pathophysiology for eosinopenia in COVID-19 remains unclear, but it may be due to inhibition of eosinophil egress from the bone marrow, reduced expression of chemokine receptors/adhesion factors, and direct eosinophil apoptosis by type 1 interferons during the acute infection. ${ }^{38}$

There were no significant differences in Monocyte count in both groups. This was in concordance with other studies however, a Korean study by GaEun Park, Cheol-In Kang, et al showed monocytopenia in the COVID-19 group compared to the control group.

\section{Neutrophil lymphocyte ratio (NLR)}

NLR was significantly raised in ICU patients as compared to Non-ICU patients, proving it to be a valuable severity indicator. Other studies also found that NLR was a useful predictive factor for critical illness probability. ${ }^{8,41}$ In another study by Jingyuan Liu, ${ }^{24}$ its was found that in the population of age $\geq 50$ years, patients with NLR $>3.13$ were prone to develop severe illness. In a study by Forget et al., it was identified that normal NLR values in a healthy adult should be between 0.78 and 3.53. In an Indian study based in Noida, similar findings were observed. ${ }^{43}$ All these studies suggested that the NLR was the most useful prognostic factor affecting the prognosis for severe illness patients. ${ }^{35}$

\section{Platelets}

In the present study, there was no significant difference observed in the platelet count. However, many other studies suggest thrombocytopenia be a predominant feature in severe COVID-19 patients. $^{8}$ Although, few studies 23 did not indicate any differences in platelet count between patients with severe disease and those exhibiting mild disease. Despite the differences in individual observations made by the authors, recent studies suggest coagulopathy as a known complication of COVID-19. Severe cases may be at risk of developing thrombocytopenia, so it is advisable to monitor the platelet count in all ICU cases. ${ }^{34}$

\section{Platelet Lymphocyte Ratio (PLR)}

The ratio of Platelet count to absolute lymphocyte count was not found to be having a significant difference while comparing both groups. In one study, PLR emerged as an independent prognostic factor for prolonged hospitalization in the multivariate analysis. ${ }^{24}$ It was suggested that a high PLR may indicate a more pronounced cytokine storm due to enhanced platelet activation. In a study conducted by Tiwari ${ }^{33}$ no significant differences in PLR were seen, as observed in the present study. However, as the platelets are dynamic parameter, the relevance of PLR can only be interpreted if follow up samples at different time points are taken.

\section{Peripheral smear findings}

Significant Peripheral smear findings in the form of reactive changes in neutrophils, lymphocytes and monocytes were observed in few ICU cases as described above. Similar morphological features were noted in the study done in Ludhiana, India. ${ }^{35}$ This can be attributed to the viral cytopathic effect of SARS-CoV.

\section{Limitation of the study}

We took the above parameters as a one-time value i.e. during admission to the Wards and after shifting to ICU. To access the severity, it's better suggested to take multiple samples at regular intervals to demonstrate a trend.

\section{CONCLUSION}

The confirmatory diagnosis of COVID-19 requires RT-PCR analysis, which is time-consuming and less accessible test. Therefore, for initial diagnosis, as well as decision-making for deciding severity and ICU requirement, certain haematological parameters are valuable. We observed lymphopenia, eosinopenia, neutrophilia, leucocytosis and decline of haemoglobin in COVID-19 confirmed cases. All these parameters showed significant aggravation in critically-ill ICU patients as compared to the non-ICU group. Increased NLR identified as an important risk factor of severe illness in pa- 
tients with SARS-CoV-2 infection and could be used as a basic haematological tool in deciding the prognosis. A onetime PLR is not indicative of disease progression. The morphology of leucocytes showing viral effects can be readily identified on peripheral smear and can be easily and serially monitored, which could also aid in diagnosis and prognostication. The effects of SARS-CoV-2 on hematopoiesis are still poorly understood, which deserves further exploration.

\section{ACKNOWLEDGEMENTS}

We are deeply grateful to all the healthcare workers in our hospital who are fighting bravely against this pandemic.

\section{Disclosure of Conflict of interest:}

The authors have no potential conflicts of interest to disclose.

\section{Source of funding}

Since it was a retrospective study requiring medical report data, no funding was needed.

\section{REFERENCES}

1. Cheng VC, Lau SK, Woo PC, Yuen KY. Severe acute respiratory syndrome coronavirus as an agent of emerging and reemerging infection. Clin Micr Rev 2007;20(4):660-694.

2. WHO. Clinical management of severe acute respiratory infection when novel coronavirus $(2019-\mathrm{nCoV})$ infection is suspected: interim guidance, 28 January 2020.

3. Ling L, Taisheng L. Interpretation of the New Health Coronary Virus Infection Pneumonia Diagnosis and Treatment Program (Trial Version 5). Chin Med J 2020;100(03):E001-E001.

4. Huang C, Wang Y, Li X, Ren L, Zhao J, Hu Y, et al. Clinical Features of Patients Infected with 2019 Novel Coronavirus in Wuhan, China. Lancet 2020;395:497-506.

5. Lippi G, Mattiuzzi C. Hemoglobin Value May Be Decreased in Patients with Severe Coronavirus Disease 2019. Hematol Transfus Cell Ther 2020;42:116-117.

6. Holshue ML, DeBolt C. Washington State 2019-nCoV Case Investigation Team First case of 2019 novel coronavirus in the United States. N Engl J Med 2020;382(10):929-936.

7. Chen NS, Zhou M. Epidemiological and clinical characteristics of 99 cases of 2019novel coronavirus pneumonia in Wuhan, China: a descriptive study. Lancet 2020;395(10223):507-513.

8. Guan WJ, Ni ZY, Hu Y, Liang WH, Ou CQ, He XJ, et al. Clinical characteristics of coronavirus disease 2019 in China. N Engl J Med 2020;382:1708-1720.

9. Zhou YG, Fu BQ, Zheng XH, Wang DS, Zhao CC, Qi YJ, et al. Aberrant pathogenic GM-CSF+ T cells and inflammatory $\mathrm{CD} 14+\mathrm{CD} 16+$ monocytes in severe pulmonary syndrome patients of a new coronavirus. bioRxiv. 2020. https://doi. org/10.1101/2020.02.12.945576

10. Henry BM. COVID-19, ECMO, and lymphopenia: a word of caution. Lancet Respir Med 2020;8(4):e24.

11. Sarkar M, Rajta P, Khatana J. Anemia in Chronic obstructive pulmonary disease: Prevalence, pathogenesis, and potential impact. Lung India 2015;32(2):142.
12. Jelkmann W. Proinflammatory Cytokines Lowering Erythropoietin Production. J Interferon Cytokine Res 1998;18(8):555-559.

13. Means RT, Krantz SB. Inhibition of human erythroid colonyforming units by interferons alpha and beta: differing mechanisms despite shared receptor. Exp Hematol 1996;24(2):204208.

14. McCranor BJ, Kim MJ, Cruz NM, Xue Q-L, Berger AE, Walston JD, et al. Interleukin-6 directly impairs the erythroid development of human TF-1 erythroleukemic cells. Blood Cells Mol Dis 2014;52(2-3):126-133.

15. Dinga X, Yua Y. Dynamic profile and clinical implications of haematological parameters in hospitalized patients with coronavirus disease 2019. Clin Chem Lab Med 2020;58(8):1365-1371.

16. Al-Tawfiq JA, Hinedi K, Ghandour J, Khairalla H, Musleh S, Ujayli A, Memish ZA. Middle East respiratory syndrome coronavirus: a case-control study of hospitalized patients. Clin Infect Dis 2014;59:160-165.

17. Qin C, Zhou L, Hu Z, Zhang S, Yang S, Tao Y, et al. Dysregulation of the immune response in patients with COVID-19 in Wuhan, China Clin Infect Dis 2020: ciaa248.

18. Usul E, San I, Bekgo B, Sahin A. The role of haematological parameters in COVID-19 patients in the emergency room. Biomark Med 2020:10.2217/bmm-2020-0317.

19. Chang D, Lin M, Wei L, Xie L, Zhu G, Dela Cruz CS, Sharma L. Epidemiologic and Clinical Characteristics of Novel Coronavirus Infections Involving 13 Patients OutsideWuhan, China. JAMA 2020;323:1092-1093.

20. Lin L, Lu L, Cao W, Li T. Hypothesis for Potential Pathogenesis of SARS-CoV-2 Infection-A Review of Immune Changes in Patients with Viral Pneumonia. Emerg Microbes Infect 2020;9:727-732.

21. Chau VQ, Oliveros E, Mahmood K, Singhvi A, Lala A, Moss N, Parikh A. The Imperfect Cytokine Storm: Severe COVID-19 with ARDS in Patient on Durable LVAD Support. J Am Case Rep 2020;34(10):72-79.

22. Wang D, Hu B, Hu C, Zhu F, Liu X, Zhang J, et al. Clinical Characteristics of 138 Hospitalized Patients With2019 Novel Coronavirus-Infected Pneumonia in Wuhan, China. JAMA 2020;12(4):1023-1025.

23. Liu J-Y, Liu Y, Xiang P, Pu L, Xiong H-F, Li C-S, et al. Neutrophil-to-lymphocyte ratio predicts severe illness patients with 2019 novel coronavirus in the early stage. MedRxiv $2020 \mathrm{htt}-$ ps://doi.org/10.1101/2020.02.10.20021584.

24. Fan BE, Chong VCL, Chan SSW, Lim GH, Lim KGE, Tan GB, et al. Hematologic parameters in patients with COVID-19 infection. Am J Hematol 2020;5(3):345-367.

25. Wu CM, Chen XY, Cai YP, Xia JA, Zhou X, Xu S, et al. Risk factors associated with acute respiratory distress syndrome and death in patients with coronavirus disease 2019 pneumonia in Wuhan, China. JAMA 2020;3(2):145-149.

26. Wong RS, Wu A, To KF, Lee N, Lam CW, Wong CK, et al. Haematological manifestations in patients with severe acute respiratory syndrome: retrospective analysis. Br Med J 2003;326(7403):1358-1362

27. Zhang JJ, Dong X, Cao YY, Yuan YD, Yang YB, Yan YQ, et al. Clinical characteristics of 140 patients infected with SARSCoV-2 in Wuhan, China. Allergy 2020;3:1-12.

28. Liu K, Fang YY, Deng Y, Liu W, Wang MF, Ma JP, et al.Clinical Characteristics of Novel Coronavirus Cases in Tertiary Hospitals in Hubei Province. Chin Med J 2020;133:1025-1031.

29. Xu XW, Wu XX, Jiang XG, Xu KJ, Ying LJ, Ma CL. Clinical Findings in a Group of Patients Infected with the 2019 Novel Coronavirus (SARS-Cov-2) Outside of Wuhan, China: Retrospective Case Series. Br Med J 2020;368:m606. 
30. Tan L, Wang Q, Zhang D, Ding J, Huang Q. Lymphopenia Predicts Disease Severity of COVID-19: A Descriptive and Predictive Study. Signal Transduct Target Ther 2020;5:33.

31. Wang X, Xu W, Hu G, Xia S, Sun Z, et al. SARS-CoV-2 Infects T Lymphocytes Through Its Spike Protein-Mediated Membrane Fusion. Cell Mol Immunol 2020;5:1-3.

32. Zheng M, Gao Y, Wang G, Song G, Liu S, Sun D. Functional Exhaustion of Antiviral Lymphocytes in COVID-19 Patients. Cell Mol Immunol 2020;17:533-535.

33. Yao Z, DuBois DC, Almon RR, Jusko WJ. Pharmacokinetic/ pharmacodynamic Modeling of Corticosterone Suppression and Lymphocytopenia by Methylprednisolone in Rats. J Pharm Sci 2008;97:2820-2832

34. Veronese N, Demurtas J, Yang L, Tonelli R. Use of Corticosteroids in Coronavirus Disease 2019 Pneumonia: ASystematic Review of the Literature. Front Med (Lausanne) 2020;7:170.
35. Li Q, Ding X, Xia G. A simple laboratory parameter facilitates early identification of COVID-19 patients. medRxiv 2020. doi:10.1101/2020.02.13.20022830

36. Lindsley AW, Schwartz JT, Rothenberg ME. Eosinophil responses during COVID-19 infections and coronavirus vaccination. J Allergy Clin Immunol 2020;S0091-6749(20)30569-8.

37. Park GE, Kang CI. Differential Cell Count and CRP Level in Blood as Predictors for the Middle East Respiratory Syndrome Coronavirus Infection in Acute Febrile Patients during Nosocomial Outbreak. J Korean Med Sci 2017;32:151-154.

38. Liu Y, Yang Y, Zhang C. Clinical and biochemical indexes from 2019-to infected patients linked to viral loads and lung injury. Sci China Life Sci 2020;63(3):364-374.

39. Singh A, Sood N, Narang V, GoyalA.Morphology of COVID-19-affected cells in peripheral blood film Bri Med J Case Rep 2020;13:e236117. 